\title{
Comparison of the efficacy between conventional moxibustion and smoke-free moxibustion on knee osteoarthritis: study protocol of a randomized controlled trial
}

Lin-lin Zhu', Jian-ying Zhou ${ }^{\dagger}$, Ling Luo ${ }^{\dagger}$, Xiang Wang, Jia-xi Peng, Sha-sha Chen, Hai-Yan Yin, Qiao-Feng Wu, Cheng-shun Zhang, Peng LV, Yong Tang ${ }^{*}$ and Shu-guang Yu*

\begin{abstract}
Background: Conventional moxibustion is a representative non-drug intervention in traditional Chinese medicine, and it has been reported to produce encouraging results and benefits in relieving symptoms and improving the quality of life for patients with knee osteoarthritis (KOA) in previous clinical trials and systematic reviews. Given that increasing concerns on the safety of generated smoke from conventional moxibustion have received much attention, smoke-free moxibustion is regarded as a potential alternative. However, whether smoke-free moxibustion would display a similar efficacy to that of conventional moxibustion still remains unclear. Therefore, this randomized controlled trial attempts to investigate the difference of efficacy between conventional moxibustion and smoke-free moxibustion in patients with KOA.

Methods/design: This is a multicenter, randomized, single-blinded, parallel-group clinical trial. A total of 138 eligible participants with KOA will be randomly allocated to two groups (conventional moxibustion group and smoke-free moxibustion group) in seven hospitals in China. Participants will receive 12 sessions of moxibustion treatment at three acupoints (EX-LE4, ST35, and ST36) over a period of 4 weeks (3 sessions per week). A smokeremoving device is placed at the top of the moxibustion device for the smoke-free moxibustion group $(n=69)$, while the conventional moxibustion group $(n=69)$ is treated with traditional moxibustion. The primary outcome measure will be the change of the global scale of the Western Ontario and McMaster Universities Osteoarthritis Index (WOMAC) from the baseline to 4 weeks. Secondary outcomes include the visual analog scale VASand Patient Global Assessment scores. Follow-up measurements will be performed on the 8th and 12th weeks after random allocation.
\end{abstract}

Discussion: This study will contribute to providing a solid foundation for the selection of moxibustion in clinical application as well as future research in moxibustion therapy.

Trial registration: ClinicalTrials.gov, NCT02772055. Registered on 12 May 2016.

Keywords: Moxa smoke, Moxibustion, Knee osteoarthritis, Randomized controlled trial, Protocol

\footnotetext{
* Correspondence: tangyong@cdutcm.edu.cn; ysg@cdutcm.edu.cn

${ }^{\dagger}$ Equal contributors

Chengdu University of Traditional Chinese Medicine Chengdu, Sichuan, China
}

\section{$\int$ Biomed Central}

(c) The Author(s). 2017 Open Access This article is distributed under the terms of the Creative Commons Attribution 4.0 International License (http://creativecommons.org/licenses/by/4.0/), which permits unrestricted use, distribution, and reproduction in any medium, provided you give appropriate credit to the original author(s) and the source, provide a link to the Creative Commons license, and indicate if changes were made. The Creative Commons Public Domain Dedication waiver (http://creativecommons.org/publicdomain/zero/1.0/) applies to the data made available in this article, unless otherwise stated. 


\section{Background}

Knee osteoarthritis (KOA) is the most common form of arthritis and is a major cause of disability and limitation of activity, especially in elderly patients [1]. It is characterized by pain and functional limitations, leading to a reduction in quality of life [2]. Osteoarthritis of the knee makes a significant impact on society, with more than 20 million Americans and 35 to 40 million Europeans developing this condition in their lifetime $[3,4]$. Some recent international guidelines advocate non-pharmacological care for patients with knee pain [5-7]. As a representative non-drug intervention in traditional Chinese medicine (TCM) and a form of acupuncture [8-10], moxibustion has been reported to produce encouraging results and benefits in relieving symptoms and improving the quality of life for patients with KOA in many studies, including clinical trials and systematic reviews [11-15].

The mechanism of moxibustion is mainly related to its thermal effects, radiation effects, the pharmacological activity of moxa, and its combustion products (volatile oil, brown tar-like substances, and moxa smoke). Moxa smoke is a primary combustion product of moxibustion, and its potential effect on the health and environment may be controversial [16]. It was reported that moxa smoke has antibacterial and antiviral effects effective in treating various conditions including wound infections, vaginal itching, uterine prolapse, anal fistulas, common warts, and so forth [17-22]. However, some studies demonstrated that high levels of monoaromatic hydrocarbons, formaldehyde, and polycyclic aromatic hydrocarbons in the moxibustion room may have adverse effects on human health [23-25]. Epidemiological investigation also showed that the moxa smoke induced a variety of undesirable reactions such as dry throat, dry eye, and coughing [26]. Furthermore, with their increased environmental awareness, people have started to question the safety of the smoke produced by burning moxa [27-29]. Because of concerns as to the potential toxicity of the smoke, many acupuncturists prefer to use a smoke purification device to remove moxa smoke during the moxibustion treatment, but few studies have reported the difference of clinical efficacy between conventional moxibustion and smoke-free moxibustion (moxibustion using the smoke-removing device).

Thus, we are currently conducting a randomized controlled clinical trial to compare the efficacy of conventional moxibustion and smoke-free moxibustion for patients with KOA.

\section{Methods/design}

\section{Design}

This is a multicenter, randomized, single-blinded, parallelgroup design clinical trial conforming to Consolidated
Standards of Reporting Trials (CONSORT) and Standards for Reporting Interventions in Clinical Trials of Acupuncture (STRICTA) guidelines [30, 31]. A Standard Protocol Items: Recommendations for Interventional Trials (SPIRIT) checklist is provided in Additional file 1. A total of 138 eligible participants with KOA will be randomly allocated into two groups (moxibustion group or smoke-free moxibustion group) with a 1:1 allocation ratio. Eligible participants who meet the clinical criteria for KOA formulated by the American College of Rheumatology (ACR) will be enrolled in this trial [32]. Seven clinical research centers in China will participate in this trial: Central Hospital of ZiBo, Chengdu First People's Hospital, Sichuan Second Hospital of TCM, Pi County People's Hospital, Xinjin County TCM Hospital, Qionglai TCM Hospital, and Nanjing Hospital of TCM. The study period is 13 weeks, with a 1 -week baseline period, 4-week treatment phase, and 8-week followup phase. After randomization, eligible participants will receive 12 sessions of moxibustion treatment over a period of 4 weeks. Patients will be assessed at the baseline visit, as well as at 2 weeks, 4 weeks, 8 weeks, and 12 weeks after allocation. The trial diagram demonstrates the procedures of the study (see Fig. 1). Table 1 outlines the measures used throughout this trial.

\section{Inclusion criteria}

Participants meeting the following criteria will be included:

- Aged between 40 and 75 years

- Diagnosed with KOA formulated by the American College of Rheumatology (ACR)

- Average severity of knee pain no more than 7 points on a visual analog scale (VAS) for most days during the past month

- Agreed to not use paregoric during the whole treatment phase

- Willingness to participate in a randomized study and to sign the informed consent form.

\section{Exclusion criteria}

Participants will be excluded if they have any of the following conditions:

- Pain in the knee that may be caused by inflammatory, malignant, or autoimmune disease or by traumatic injury

- Serious diseases including cancer, uncontrolled hypertension, diabetes mellitus requiring insulin injection, life-threatening cardiovascular or neurological events, chronic respiratory disease, a hemorrhagic disorder, or serious mental diseases 


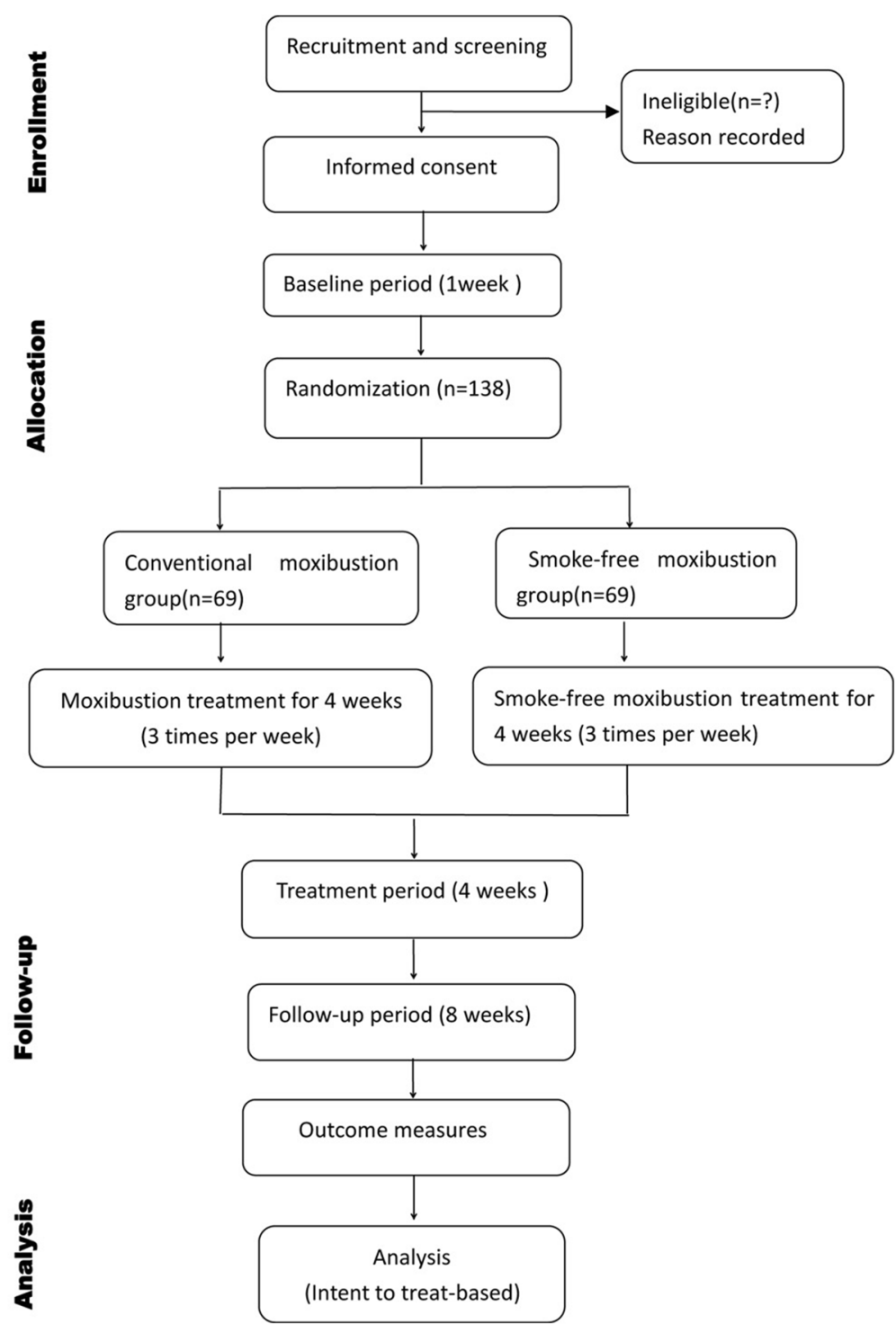

Fig. 1 Flowchart of the study process. Participants with a diagnosis of KOA will be recruited from seven centers. All project general practitioners (GPs) were trained for 1 day. The trial period will consist of 12 sessions of moxibustion treatment over a period of 4 weeks. The treatment frequency will be three sessions per week, and there will be a 2-month follow-up period. The outcomes will be measured at every visit

- Knee replacement surgery, arthroscopy of the affected knee within the past year, steroid or hyaluronic acid injection in the knee joints within the previous 3 months

- Life-threatening cardiovascular or neurological events within the past year

- Physiotherapy or other treatments for osteoarthritis knee pain (with the exception of non-steroidal anti-inflammatory drugs) during the previous 4 weeks

- Current participation in another clinical trial

- Accepted acupuncture, moxibustion, cupping, or herbal medicine within the past 4 weeks.

\section{Sample size calculation}

A power analysis has been undertaken before conducting this trial for further calculation. The significance level is 0.05 , and the statistical power is 0.90 , consistent with a previous trial on moxibustion for $\mathrm{KOA}$ and our pilot study [33]. To compensate for an anticipated dropout rate of 15\%, 138 patients with KOA (69 in each group) will be recruited from seven centers in China.

\section{Recruitment strategies}

There will be three strategies for KOA participant recruitment. First, participants will be recruited from the outpatient and inpatient departments of the seven 
Table 1 Study design schedule

\begin{tabular}{|c|c|c|c|c|c|}
\hline \multirow[t]{3}{*}{ Period } & \multirow{2}{*}{$\begin{array}{l}\text { Inclusion } \\
\text { Baseline }\end{array}$} & \multicolumn{2}{|l|}{ Treatment } & \multicolumn{2}{|l|}{ Follow-up } \\
\hline & & First assessment & Second assessment & Third assessment & Fourth assessment \\
\hline & 0 week & 2 weeks after inclusion & 4 weeks after inclusion & 8 weeks after inclusion & 12 weeks after inclusion \\
\hline Inclusion confirmed & $\sqrt{ }$ & & & & \\
\hline Informed consent & $\sqrt{ }$ & & & & \\
\hline Randomization and blinding & $\sqrt{ }$ & & & & \\
\hline Sociodemographic characteristics & $\sqrt{ }$ & & & & \\
\hline Disease history & $\sqrt{ }$ & & $\sqrt{ }$ & & \\
\hline Treatment history & $\sqrt{ }$ & & $\sqrt{ }$ & & \\
\hline Comorbidity & $\sqrt{ }$ & & $\sqrt{ }$ & $\sqrt{ }$ & $\sqrt{ }$ \\
\hline Current treatment & $\sqrt{ }$ & & $\sqrt{ }$ & $\sqrt{ }$ & $\sqrt{ }$ \\
\hline \multicolumn{6}{|l|}{ Outcomes } \\
\hline WOMAC & $\sqrt{ }$ & $\sqrt{ }$ & $\sqrt{ }$ & $\sqrt{ }$ & $\sqrt{ }$ \\
\hline VAS & $\sqrt{ }$ & $\sqrt{ }$ & $\sqrt{ }$ & $\sqrt{ }$ & $\sqrt{ }$ \\
\hline PGA & & $\sqrt{ }$ & $\sqrt{ }$ & $\sqrt{ }$ & $\sqrt{ }$ \\
\hline \multicolumn{6}{|l|}{ Trial evaluation } \\
\hline Outcome analysis & & & & & $\sqrt{ }$ \\
\hline Adverse event & & & $\sqrt{ }$ & & \\
\hline Causes of dropout & & & $\sqrt{ }$ & & \\
\hline Safety analysis & & & $\sqrt{ }$ & & \\
\hline Compliance analysis & & & $\sqrt{ }$ & & \\
\hline
\end{tabular}

WOMAC Western Ontario and McMaster Universities Osteoarthritis Index, VAS visual analog scale, PGA Patient Global Assessment

hospitals. Second, printed recruitment posters will be distributed to local communities. Finally, advertisements will be posted in local newspapers and through the website.

\section{Random assignment}

Each eligible subject will be randomly assigned to either the conventional moxibustion group or the smoke-free moxibustion group. A random number list will be generated with SPSS 16.0 (SPSS Inc., Chicago, IL, USA) by a statistician who is not directly involved with the study. The researchers in each clinical research center will call the independent statistician at the center for the randomized number, and the statistician will be immediately informed of the participant's randomized number and treatment group by mobile message. The independent researcher will record the patient's details and the number assigned to that patient. Central randomization has strict limits of authority: no one can check the files except the top principal investigator.

\section{Blinding}

This is a single-blinded trial; participants are unaware of the assignments. Participants will be separated and treated in two different rooms. Meanwhile, the assessors of therapeutic effect, the data manager, and the statistician will be kept blinded to the treatment allocation. To prevent accidental un-blinding, there will be no contact between the study team and the subjects until after the data cleaning, database lockdown, and analysis are complete.

\section{Interventions}

The treatment will be applied in 12 sessions, three times per week for 4 weeks. The conventional moxibustion group and the smoke-free moxibustion group will receive moxibustion at three standard acupuncture points: Neixiyan (EX-LE4), Dubi (ST35), and Zusanli (ST36) [11, 33] (Fig. 2). The selection of acupoints is based on TCM meridian theory to treat knee joint pain, known as the "Bi syndrome," and on some similar studies [11, 12, 34-36]. All project general practitioners (GPs) have completed at least 5 years of training in acupuncture and moxibustion and are registered as Chinese medicine practitioners by the National Health and Family Planning Commission of the People's Republic of China. Project GPs were trained for 1 day by an investigator who is an experienced medical acupuncturist, to standardize all aspects of the treatment protocol. To ensure implementation of the study, the investigator must maintain regular contact with the GPs to answer and settle any treatment-related problems. Each GP was provided with a detailed treatment manual describing administration of the moxibustion interventions as well as the trial 


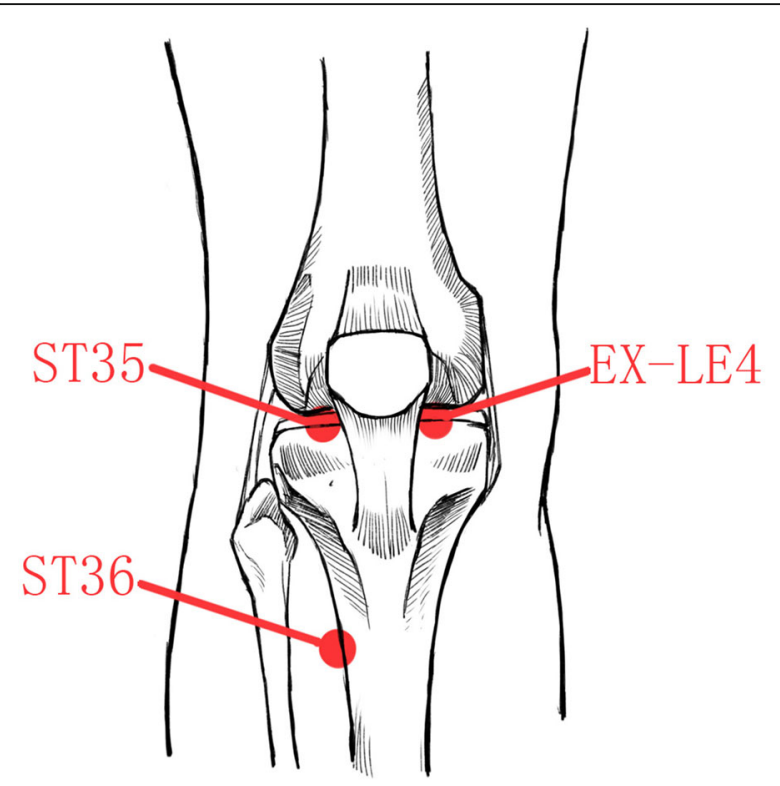

Fig. 2 Acupoints used in the trial. The lines refer to the acupoints Dubi (ST35) and Zusanli (ST36) on the lateral side of the knee and Neixiyan (EX-LE4) on the medial side of the knee

protocol. Two separated moxibustion rooms were provided for the different groups by the centers, doors and windows will be opened in the treatment process. The participants are usually required to wear loose clothing to expose the knee joints and to maintain a comfortable sitting position for treatment.

\section{Moxibustion group (group A)}

The moxibustion group will use a moxa device (Yijiu moxa device, Maanshan, Anhui, China) at the acupoints (EX-LE4, ST35, ST36). The moxa pillar is cylindrical, 1.5 $\mathrm{cm}$ in diameter, and $3 \mathrm{~cm}$ long. A lighted moxa pillar will be put into the moxa device attached at each acupoint, and the therapist will make sure the patient feels a warm but not scorching sensation (Fig. 3). One

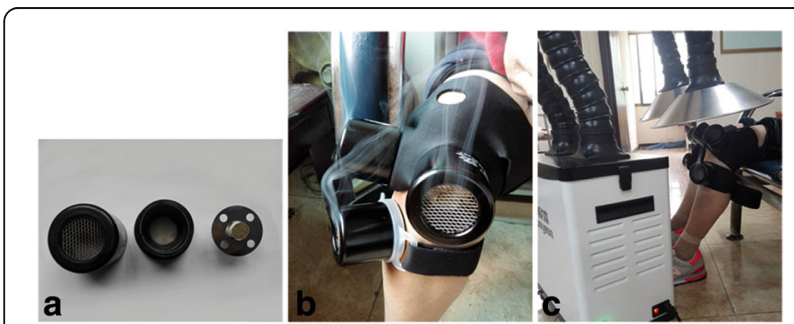

Fig. 3 Diagram of the moxibustion and smoke-free moxibustion devices. a This new moxa device is composed of three parts. b Participants are treated at acupoints Dubi (ST35), Zusanli (ST36), and Neixiyan (EX-LE4) with devices consisting of a moxa pillar. The moxa device has an opening in the center that allows heat and smoke from the burning pillar to stimulate the acupoints. $\mathbf{c}$ In the smoke-free moxibustion group, investigators place a purification device at the top of the moxibustion device to remove the moxa smoke acupoint is treated for $30 \mathrm{~min}$ each time. Three acupoints are treated by moxibustion at the same time.

\section{Smoke-free moxibustion group (group B)}

In contrast to the moxibustion group, a specifically designed device (Shenzhen Conyson Electronic Technology Co, Ltd., C200 moxa smoke purification device, Shenzhen, China) is applied to remove the moxa smoke for the smoke-free group in the process of moxibustion. Otherwise, the selection of acupoints and other interventions in both groups must be the same (Fig. 3).

\section{Outcome assessments}

\section{Primary outcome measurement}

The primary outcome measure will be the mean change in the global scale value of the Western Ontario and McMaster Universities Osteoarthritis Index (WOMAC) from baseline to 4 weeks [37]. The WOMAC, one of the most widely used arthritis assessment tools, is composed of 24 questions to assess disability related to osteoarthritis. It includes three subscales to measure pain (5 questions), stiffness (2 questions), and physical function (17 questions) regarding $\mathrm{KOA}$; higher scores indicate more severe impairment. All 24 questions will be listed in a numerical rating scale ranging from 0 (no symptoms) to 3 (maximum symptoms). If both knees are affected, the more severe knee will be assessed. Physicians who are blinded to the treatment allocation will help patients perform the self-assessment and collect and record the total score.

\section{Secondary outcome measurements}

The secondary outcomes include the mean changes in the VAS and Patient Global Assessment (PGA) scores. The pain intensity of KOA will be assessed using a 100 mm VAS (0, absence of pain; 100, the worst pain imaginable). The pain VAS is a standard tool in chronic pain studies for measuring pain intensity. We will evaluate the mean change in the VAS at baseline ( 0 week) and at $2,4,8$, and 12 weeks after allocation. The PGA score is a self-reported 5-point measurement used to evaluate overall improvement after treatment. It has been used in various studies to assess therapeutic effects [38-40]. Participants individually evaluate their improvement by selecting one of 5 options (much improved, minimally improved, no change, minimally worse, or much worse) at $2,4,8$, and 12 weeks from the baseline.

\section{KOA diary}

Subjects will be provided with KOA diaries to record the specific time of knee pain attacks, the associated phenomena, and any medication used. This information will be recorded every day throughout the study period. All 
patients will be carefully instructed on how to use this diary to rate their symptoms.

\section{Statistical analysis plan Data integrity}

The research will ensure that no record will be missed or omitted in the original data source. Any corrections should be explained in the appended notes signed and dated by the physicians participating in the clinical trial. The primary input of the data is not permitted to be changed. After the observation of a clinical case, a case report form (CRF) will be submitted to the project directors for verification. The principal investigators will have full access to the data. The data manager will clarify any questions regarding the CRF with the researchers via clinical supervisors. Researchers should answer any questions as soon as possible so that the data manager will be able to conduct modification, validation, and input of the data. The data monitoring committee comprises a medical statistician and an epidemiologist, who are independent from the investigators and sponsors, for source data organizing, coding, and range checking for data values to ensure data quality.

\section{Analysis}

The primary analysis of the data will be undertaken using the intention-to-treat principle with all randomized patients included in the analyses. A multiple imputation adjustment approach will be used for the missing data. Demographic and baseline data will be analyzed with standard, descriptive statistics. Between-group differences will be tested using repeated measure analyses of variance. The accepted level of significance for all analyses is $P<0.05$. The whole data analysis process will be performed by statisticians who are independent from the research team and blinded to the group settings. SPSS software (SPSS 16.0, SPSS Inc., Chicago, IL, USA) will be used for the data analysis.

\section{Safety monitoring}

Possible adverse events (AEs) resulting from moxibustion include blisters, redness, itching, and burns. All unexpected and unintended responses will be reported as AEs by the researcher at every visit. AEs will be carefully recorded in the CRF by the corresponding research staff.

\section{Discussion}

Moxibustion is widely used in Asian countries to treat various disorders and is reported to be effective for relieving the pain and functional limitations of KOA [10, $12,13]$. Recent research has focused on the composition and the safety of moxa smoke, but few studies have considered the effect of moxibustion with removal of the moxa smoke [25]. The aim of this trial is to compare the effectiveness of conventional moxibustion and smoke-free moxibustion for patients with KOA.

In clinical application, the smoke-free moxa sticks have been used because of the concerns about moxa smoke; however, some experienced acupuncturists consider that its clinical efficacy may not be equal to that of conventional moxibustion. In the present study, a special device removing the moxa smoke will not be used in the conventional moxibustion group but only in the smokefree group, and the same kind of moxa sticks will be chosen in these two groups to ensure that other conditions except for the moxa smoke reach consensus. The study aims to explore the influence on effectiveness of removing moxa smoke in the process of moxibustion. The device to remove the moxa smoke has been used in clinical practice, and our design is based on clinical application.

Because of the objective of this trial to clarify whether removing moxa smoke may be interfering with the clinical efficacy of conventional moxibustion, and the difficulty of using the double-blind methodology in acupuncture and moxibustion, we designed a singleblind study; therefore, it is not necessary to set up a wait-list group. The treatment acupoints and intervention period are based on some positive result studies and clinical experience in order to ensure their effectiveness $[11-13,15]$.

The design and methodological rigor of this trial will allow for collection of high-quality data to compare the effectiveness of conventional moxibustion and smokefree moxibustion for patients with KOA. Thus, the trial will contribute to providing a selection of moxibustion in practice, as well as promote the clinical application of moxibustion therapy.

\section{Trial status}

This study is currently in the recruitment phase. Participant recruitment started in June 2016 and is expected to end in May 2017.

\section{Additional file}

Additional file 1: SPIRIT 2013 checklist: recommended items to address in a clinical trial protocol and related documents. (DOC $119 \mathrm{~kb}$ )

\section{Abbreviations}

ACR: American College of Rheumatology; AE: Adverse event; CRF: Case report form; GP: General practitioner; KOA: Knee osteoarthritis; TCM: Traditional Chinese medicine; VAS: Visual analog scale; WOMAC: Western Ontario and McMaster Universities Osteoarthritis Index

\section{Acknowledgements}

The authors would like to acknowledge the cooperation of the participants in this trial. 


\section{Funding}

This trial was financially supported by the 973 Program of China (2015CB554504), Sichuan Provincial Innovative Research Team Program (2014TD0018, 2015TD0010), and Innovative Research Team at the University of Sichuan Province (16TD0015)

\section{Availability of data and materials}

Not applicable.

\section{Authors' contributions}

ZLL, ZJY, and LL contributed equally to this work. ZLL, ZJY, and LL wrote the study protocol and drafted this manuscript. TY, WQF and ZCS made a substantial contribution to the study protocol design. LP and WX participated in the qualitative study design and in the critical revision. PJX and YHY participated in the critical revision of the manuscript. CSS helped to draft the manuscript. TY and YSG had final responsibility for the decision to submit for publication. All authors read and approved the final manuscript.

\section{Authors' information}

Not applicable.

\section{Competing interests}

The authors declare that they have no competing interests.

\section{Consent for publication}

Consent for publication from the participants to report individual patient data has been obtained from each participant.

\section{Ethics approval and consent to participate}

The following ethical review committees have reviewed and approved this study. The protocol (version 2.0, dated 17 November 2015) was approved by the IRB/EC of the Teaching Hospital of Chengdu University of TCM (reference: 2015KL-014). It also applies to these hospitals: Chengdu First People's Hospital, Sichuan Second Hospital of TCM, Pi County People's Hospital, Xinjin County TCM Hospital, Qionglai TCM Hospital, and Central Hospital of ZiBo. The Ethics Committee of Nanjing Hospital of TCM approved the study (reference number KY2016019).This trial will be conducted according to the principles of the Declaration of Helsinki (version Edinburgh 2000). All participants will be informed about the purpose of the trial and its risks and benefits, and written informed consent will be obtained by the local study coordinator prior to entry into the trial.

\section{Publisher's Note}

Springer Nature remains neutral with regard to jurisdictional claims in published maps and institutional affiliations.

\section{Received: 7 August 2016 Accepted: 16 February 2017}

\section{Published online: 24 April 2017}

References

1. Dawson J, Linsell L, Zondervan K, Rose P, Carr A, Randall T, Fitzpatrick R. Impact of persistent hip or knee pain on overall health status in elderly people: a longitudinal population study. Arthritis Rheum. 2005;53:368-74.

2. Peat G, McCarney R, Croft P. Knee pain and osteoarthritis in older adults: a review of community burden and current use of primary health care. Ann Rheum Dis. 2001:60(2):91-7.

3. Felson DT, Naimark A, Anderson J, Kazis L, Castelli W, Meenan RF. The prevalence of knee osteoarthritis in the elderly. The Framingham Osteoarthritis Study. Arthritis Rheum. 1987;30:914-8.

4. Mannoni A, Briganti MP, Di Bari M, Ferrucci L, Costanzo S, Serni U, Masotti G, Marchionni N. Epidemiological profile of symptomatic osteoarthritis in older adults: a population based study in Dicomano, Italy. Ann Rheum Dis. 2003; 62.576-8 doi:10.1136/ard.62.6.576.

5. Osteoarthritis: care and management in adults [clinical guideline CG177]: methods, evidence and recommendations. National Institute for Health and Care Excellence. http://www.nice.org.uk/guidance/CG177. Accessed Feb 2014.

6. McAlindon TE, Bannuru RR, Sullivan MC, Arden NK, Berenbaum F, BiermaZeinstra SM, et al. OARSI guidelines for the non-surgical management of knee osteoarthritis. Osteoarthritis Cartilage. 2014;22:363e88.
7. Zhang W, Nuki G, Moskowitz RW, Abramson S, Altman RD, Arden NK, et al. OARSI recommendations for the management of hip and knee osteoarthritis: Part III: changes in evidence following systematic cumulative update of research published through. Osteoarthritis Cartilage. 2009:2010(18):476e99.

8. Wainapel SF, Thomas AD, Kahan BS. Use of alternative therapies by rehabilitation outpatients. Arch Phys Med Rehabil. 1998;79:1003-5. doi:10.1016/S0003-9993(98)90102-2.

9. World Health Organization. WHO international standard terminologies on traditional medicine in the Western Pacific Region. Western Pacific Region: World Health Organization; 2007.

10. Tang Y, Yin HY, Rubini P, Llles P. Acupuncture-induced analgesia: a neurobiological basis in purinergic signaling. Neuroscientist. 2016;22(6):56378. 1073858416654453.

11. Zhao L, Cheng K, Wang L, Wu F, Deng H, Shen X, et al. Effectiveness of moxibustion treatment as adjunctive therapy in osteoarthritis of the knee: a randomized, double-blinded, placebo-controlled clinical trial. Arthritis Res Ther. 2014;16(3):R133.

12. Chen RX, Chen MR, Xiong J, Su TS, Zhou MQ, Sun JH, et al. Comparative effectiveness of the deqi sensation and non-deqi by moxibustion stimulation: a multicenter prospective cohort study in the treatment of knee osteoarthritis[J]. Evidence-based complementary and alternative medicine: eCAM. 2013;(1):906947.

13. Ren X, Yao C, Wu F, Li Z, Xing J, Zhang H. Effectiveness of moxibustion treatment in quality of life in patients with knee osteoarthritis: a randomized, double-blinded, placebo-controlled trial. Evid Based Complement Altern Med. 2015:2015:569523.

14. Shen X, Lao L, Zhang Y. Biophysical and clinical research on acupuncture and moxibustion. Evid Based Complement Altern Med. 2015;2015:518138.

15. Li A, Wei ZJ, Liu Y, Li B, Guo X, Feng SQ. Moxibustion treatment for knee osteoarthritis: a systematic review and meta-analysis. Medicine. 2016:95(14): e3244.

16. Deng $H$, Shen $X$. The mechanism of moxibustion: ancient theory and modern research. Evid Based Complement Altern Med. 2013;2013:379291.

17. Lu Y, Ye CM, Gao JF, Wu BO. Moxa fumigation for 575 surgical infectious conditions (annex: inhibiting bacteria test of the moxa smoke). An Hui Zhong Yi Xue Yuan Xue Bao. 1998;7(4):36-7.

18. Hitosugi N, Ohno R, Hatsukari I, Mizukami S, Nagasaka H, Matsumoto I, Sakagami $H$. Diverse biological activities of moxa extract and smoke. In vivo (Athens, Greece). 2000;15(3):249-54

19. Chen Q, Wu QF, Xin FH, Tao SH. Control observation on moxa stick fumigation and ultraviolet irradiation. Jiang Su Da Xue Xue Bao (Yi Xue Ban). 2002;12(5):523-5.

20. $\mathrm{Ma} \mathrm{HL}, \mathrm{Fu} X Y$, Tang SZ. Air sterilization comparison between moxa stick fumigation and ultraviolet irradiation. Xin Jiang Yi Xue. 2006;36(1):107-8.

21. Zhou CL, Feng XM, Wang JH, Wu LY, Zhang SJ, Chen HP, Wu HG. Research advance on moxa smoke. J Acupunct Tuina Sci. 2011;9:67-72.

22. Matsumoto T, Katai S, Namiki T. Safety of smoke generated by Japanese moxa upon combustion. Eur J Integr Med. 2016;8:414-22.

23. Zhang R. Accidents in acupuncture treatment: history and current state. J Chin Integrat Med. 2004;2:306-13.

24. Zhao YY, Li WY. Effects of moxa smoke on moxibustion doctors suffering from chronic pharyngolaryngitis. Lishizhen Med Mater Med Res. 2013;24:1801-2.

25. Mo F, Chi C, Guo M, Chu XY, Li YL, Shen XY. Characteristics of selected indoor air pollutants from moxibustion. J Hazard Mater. 2014;270:53-60.

26. Chang $Y$, Wang $X$, Yang $H$. Small sample size epidemiological survey of the time-related exposure reaction of acupuncturists under the moxa-smoke environment. J Chengdu Univ Tradit Chin Med. 2015;38(4):6-9.

27. Li CM. The influence of inhalation of moxa smoke on moxibustion doctors. Int J Tradit Chin Med. 1994;16:57-8.

28. Xu J, Deng $H$, Shen X. Safety of moxibustion: a systematic review of case reports. Evid Based Complement Altern Med. 2014:2014:783704.

29. Hsu YC, Chao HR, Shi SI. Human exposure to airborne aldehydes in Chinese medicine clinics during moxibustion therapy and its impact on risks to health. J Environ Sci Health A. 2015;50(3):260-71

30. Schulz KF, Altman DG, Moher D. CONSORT 2010 statement: updated guidelines for reporting parallel group randomised trials. BMC Med. 2010;8(1):1.

31. MacPherson $H$, Altman DG, Hammerschlag R, Li YP, Wu TX, White A, et al. Revised standards for reporting interventions in clinical trials of acupuncture (STRICTA): extending the CONSORT statement. J Evid Based Med. 2010;3(3):140-55. 
32. American College of Rheumatology Subcommittee on Osteoarthritis Guidelines. Recommendations for the medical management of osteoarthritis of the hip and knee: 2000 update. Arthritis Rheum. 2000;43:1905-15.

33. Kim TH, Kim KH, Kang JW, Lee MH, Kang KW, Kim JE, et al. Moxibustion treatment for knee osteoarthritis: a multi-centre, non-blinded, randomised controlled trial on the effectiveness and safety of the moxibustion treatment versus usual care in knee osteoarthritis patients. PLoS One. 2014; 9(7):e101973.

34. Witt C, Brinkhaus B, Jena S, Linde K, Streng A, Wagenpfeil S, et al. Acupuncture in patients with osteoarthritis of the knee: a randomised trial. Lancet. 2005:366:136-43.

35. Scharf HP, Mansmann U, Streitberger K, Witte S, Kramer J, Maier C, et al. Acupuncture and knee osteoarthritis: a three-armed randomized trial. Ann Intern Med. 2006;145:12-20.

36. Hinman RS, McCrory P, Pirotta M, lan R, Andrew F, Kay M, et al. Acupuncture for chronic knee pain: a randomized clinical trial. JAMA. 2014;312(13):1313-22.

37. Bellamy N. Validation study of WOMAC: a health status instrument for measuring clinically-important patient-relevant outcomes following total hip or knee arthroplasty in osteoarthritis. J Orthop Rheumatol. 1988;1:95-108.

38. Hoving $\mathrm{JL}$, Koes BW, de Vet HCW, van der Windt DAWM, Assendelft WJJ, van Mameren H, Deville WLJM, Pool JJM, Scholten RJPM, Bouter LM. Manual therapy, physical therapy, or continued care by a general practitioner for patients with neck pain. A randomized, controlled trial. Ann Intern Med. 2002;136:713-22

39. De Hertogh W, Vaes P, Devroey D, Louis P, Carpay H, Truijen S, Duquet W, Oostendorp R. Preliminary results, methodological considerations and recruitment difficulties of a randomised clinical trial comparing two treatment regimens for patients with headache and neck pain. BMC Musculoskelet Disord. 2009;10:115.

40. De Hertogh WJ, Vaes PH, Devroey D, Truijen S, Duquet W, Oostendorp R. Management of headache disorders: design of a randomised clinical trial screening for prognostic patient characteristics. BMC Musculoskelet Disord. 2007:8:38

\section{Submit your next manuscript to BioMed Central and we will help you at every step:}

- We accept pre-submission inquiries

- Our selector tool helps you to find the most relevant journal

- We provide round the clock customer support

- Convenient online submission

- Thorough peer review

- Inclusion in PubMed and all major indexing services

- Maximum visibility for your research

Submit your manuscript at www.biomedcentral.com/submit 